\title{
THE RELATION OF APPARENT SHAPE TO APPARENT SLANT IN THE PERCEPTION OF OBJECTS ${ }^{1}$
}

\author{
JACOB BECK AND JAMES J. GIBSON \\ Cornell University
}

The problem of how we perceive the shape of an object in space is complicated by the optical fact that to a particular retinal form there does not correspond a unique physical form but a whole set of possible physical forms. A given sheaf of light rays whose cross section is a particular form may be reflected from any surface whose margins coincide with the margins of the sheaf of rays; the surface does not have to be perpendicular but may be inclined to the sheaf. This set of surfaces is, mathematically, the family of perspective transformations of the cross section. A square cross section, for example, may arise from a family of variously inclined trapezoids. A trapezoidal cross section may arise not only from an inclined square but also from a different family of inclined trapezoids, of which the square is only a special case.

The optical fact seems to contradict the possibility of a correspondence between variations of the retinal image (the proximal stimulus) and variations of the object (the distal

\footnotetext{
1 This paper is a revision of a thesis presented for the degree of Master of Arts at Cornell University in 1951. The work was supported in part by the U. S. Air Force under Contract AF 33(038)-22373 with the Perceptual and Motor Skills Research Laboratory, Lackland Air Force Base, San Antonio, Texas. Permission is granted for reproduction, translation, publication, use, and disposal in whole and in part by or for the U. S. Government. The final article represents the joint efforts of both authors, having assumed its final form only after long discussion and many revisions. Beck collected the data and prepared the first drafts. Gibson is mainly responsible for the theoretical development and the terminology.
}

stimulus). Consequently it makes it impossible to assume any simple correspondence between the retinal image and a true perception of form. How, then, do we get an objective perception of form?

The hypothesis of a psychological invariant.-One kind of answer is to suppose that men have hypothetical sensations of form, each of which is in correspondence with the cross section of a particular sheaf of rays, and then to suppose that a sensation of form is corrected by past experience with the object in question. The difficulties with this answer have often been pointed out and need not be reviewed here. A more sophisticated answer is to suppose that we get a perception of the inclination of the form along with the sensation of the form as such, and then to suppose that these two experiences are related as mutually dependent processes-that they are, in Koffka's words, "linked together" $(9$, p. 229). This is a very appealing hypothesis to explain constancy. If it is true that an impression of shape is never obtained without a concomitant impression of slant, then it is possible that these two impressions may interact psychologically (or physiologically). If the interaction is precise, there could arise a final perception which, like the ratio (or the sum or the product) of two variable quantities, is constant. This constant product of two variable impressions might be the basis of our objectively correct perceptions of shape.

The hypothesis that impressions of 
shape and slant are coupled psychologically is implied by Helmholtz ( 6 , p. 15) and is explicitly stated by Koffka (9, p. 229). It is closely related to the hypothesis that impressions of the size and distance of an object are coupled psychologically, which has an even larger literature. This has been frequently stated and studied by Boring and has recently been reviewed by Kilpatrick and Ittelson (8), who have a neat formula for what they call the size-distance invariance hypothesis, namely: a retinal projection of given size determines a unique ratio of apparent size to apparent distance. This formula can well be applied equally to shape: $a$ retinal projection of a given form determines a unique relation of apparent shape to apparent slant. This is the explicit hypothesis with which we are concerned.

The hypothesis of optical stimulus variables for the slant of a surface.-A flat object seen under ordinary conditions always has edges and usually has a textured surface. The form of the edges and the nature of the texture are projected in the retinal images of two eyes. When the surface is inclined to the line of sight, the images manifest a compression of both form and texture (3, p. 172), and also a skew of form and texture in one image relative to the other and a similar skew in each image during head movement. These three types of compression and skew are concomitant, and are proportional to the optical slant of the surface. Conceivably they are stimuli for the impression of optical slant. Conceivably they are also stimuli for the impression of shape if we are willing to assume that the form and the texture of a surface are not separable but are closely interrelated. This possibility suggests a quite different explanation for the constancy of shape perception, namely, that it is based on constant properties of optical stimulation.

It should be noted that the compression of the texture can be eliminated from each retinal image by presenting an object with a homogeneous surface, and that the two types of skew of both form and texture can be eliminated by requiring monocular vision and a motionless head. The stimulus array is thereby reduced to the form of the edges alone. The resulting percept is a sort of disembodied form - the merest ghost of a substantial object (4). Nevertheless, this is the kind of form presupposed in current theories of constancy-textureless, single, and static. It is the "apparent shape" referred to in the formula above. It is possible, however, that the kind of form which shows constancy is textured, disparate, and mobile.

The implications of the hypothesis of a shape-slant invariant. - The formula should be examined for what it does and does not imply, with a view to experiments. It asserts first that a retinal form determines a family of possible apparent shapes, and second that each of these apparent shapes is linked with a corresponding apparent slant. An implication of the first assertion is that this reduced stimulus does not determine a single phenomenal shape, or a "sensation" of shape. It contradicts the commonly accepted view that, when the cues for depth are absent, the perceived shape of an object is determined by the shape of the retinal image. Boring, for instance, has expressed the opinion that perceived size depends on retinal size under reduction conditions $(2,7)$, and presumably the same rule is supposed to hold for shape. The formula seems to imply that there is no such thing as a retinal stimulus for shape, but only a stimulus for a family of shapes. Here is a clear-cut experimental issue: 
Does or does not phenomenal shape become variable and indeterminate when the stimulus is a retinal contour form?

It should also be noted that the formula does not say anything explicit about what the apparent shape will be when the apparent slant is determined, i.e., when the cues for depth are present. It applies to the "pure" form obtained when the cues are absent. It states that an impression of shape under the latter conditions will vary inversely with the accompanying impression of slant; that the two impressions will covary in the manner necessary to satisfy the invariant relation. If an impression of slant is somehow aroused, the apparent shape must be that of an object which would yield the current retinal image; if an impression of shape is somehow aroused, the apparent slant must be that physical slant which would yield the retinal image. This implication of the formula can also be tested experimentally. An extra hypothesis is required to account for the arousal of an impression of slant or shape: the readiest explanation would be that an assumption, attitude, or expectation determines it. In the absence of sensory information the organism presumes a slant or a shape for the object. A presumptive slant would be the frontal plane, or perhaps a slant which conforms to that of the background surface on which the object stands. A presumptive shape would be the circle within its family of ellipses, or a rectangle in the family of trapezoids.

The formula says nothing about shape perception when cues for slant are present. Since this is the condition under which shape constancy occurs, it is not in itself an explanation of constancy. Nevertheless it seems to be consistent with such facts; perhaps it is a consequence or corollary of constancy which becomes manifest only when the cues for slant are absent. The supplementary theory for the case when the cues are present might belong to either of the following types:

A follower of Helmholtz (6) would argue that the organism discovers in the course of time that the cues for the slant of an object vary concomitantly with the retinal projected form of the object. Only then is he in a position to infer the true shape of the object from the retinal shape by combining this information with the cues for slant. The psychological linkage between impressions of shape and slant would arise as the result of associations between them.

A psychophysical theory, on the other hand, would argue that if the concomitant variation referred to is a discoverable fact it must be an invariant of stimulation itself. The compressionand-skew of the dual retinal images, already referred to, is a high-order variable of stimulation which reflects both the slant and the shape of the physical object. This stimulus invariant is a matter of optics and geometry, not of psychology, and it might prove to be the cause of a simple and immediate experience of shape-at-aslant. The psychological linkage between impressions of shape and slant, if verifiable, would be manifest only when the stimulus invariant had been eliminated in the retinal images, leaving only bare contours and form. In this event, associative processes could be expected to occur and perhaps also the semi-intellectual processes of assumption, expectation, and inference described by Helmholtz.

The experiments to be reported are tests of the shape-slant invariance formula and its implications. They cannot be expected to decide between alternative theories of shape constancy but they may clarify the factual background for the theories.

\section{EXPERIMENT I}

The question is whether apparent shape is linked with apparent slant when the latter is not determined by stimulation. Although experiments by Thouless (12) and by Eissler ( 9 , p. 229) are relevant to the question in a general way, only one study exists which set out specifically to test the hypothesis, that of Stavrianos (11). She had her Os make explicit ${ }^{*}$ judg- 
ments of the shape and the slant of an inclined rectangle, separately, under three conditions of observation: (a) full binocular vision, $(b)$ binocular vision with reduction tubes, and $(c)$ monocular vision with a reduction tube. She found that the progressive elimination of stimuli for slant caused the judgments of slant to become more variable and less accurate, but not the judgments of shape. The latter did not deteriorate correspondingly and the error of seen shape did not vary as a strict function of the error of seen slant, as the hypothesis demands. The judgments of shape and of slant, however, had been made successively and separately, and this fact might explain the failure to verify the hypothesis. Two other experiments were therefore run in which an attempt was made to force $O$ to take a "shape-and-slant" attitude during both judgments. In both, a precise relation between errors of shape and slant failed to appear in the data, although they did justify the conclusion that "perception of shape may be roughly related to explicit judgments of inclination." A crucial test of the hypothesis probably requires an experiment in which the judgments of shape and slant are not explicit, but are implicit in the same act of matching the standard object. ${ }^{2}$

Another purpose of the present experiment was to discover whether, in the absence of stimuli for slant, the retinal shape determines only a family of possible perceived shapes or whether it determines the perception of a single shape in the frontal plane.

Apparatus and procedure.-The plan of the experiment was to present $O$ with a standard

2 Another test of the hypothesis has been noted since this paper was written, by J. Langdon (10). With a quite different experiment, Langdon also failed to get the sort of results which Koffka believed should follow from the invariant relationship. object suspended in darkness and have him match it for both shape and slant with one of a series of comparison objects in the light which systematically varied in shape and slant. The standard object was viewed with monocular vision and a motionless head, the comparison objects with binocular vision and a mobile head. The standard object was a luminous shape cut out of cardboard over a sheet of textureless ground glass in a light box. The latter was set at $89 \mathrm{in}$. from the eye and could be rotated on a horizontal axis. The comparison objects were a set of sheet-metal shapes, painted white and mounted at different degrees of slant (or no slant) on a black background or panel. They were set at approximately 30 in. from the eyes and were one-third the size of the standard object.

The experiment was run with two kinds of standard objects to be matched, quadrilaterals and triangles. The former included a rectangle but the latter did not include an equilateral triangle, all triangles being flatter than equilateral. The former included a "normal" shape; the latter did not.

On half the trials $O$ was presented with a physically slanted 10 - by 8 -in. rectangle, and on the other half with a physically frontal trapezoid equivalent to the cross section of the light rays from the rectangle. (The latter will be referred to as the "cross-sectional" trapezoid.) These two physically different but optically equivalent objects yielded the same distribution of judgments; hence we may conclude that they were indistinguishable, and that stimulation for slant had been effectively eliminated in viewing the standard. A similar procedure was used for the triangles, with the same outcome. The standard slanted triangle was of 10 -in. base and 8 -in. altitude.

When the rectangle was presented, the face of the light box was inclined either floorwise or ceilingwise (top backward or top forward) at three angles of inclination, $30^{\circ}, 45^{\circ}$, and $60^{\circ}$. Corresponding to these six stimuli were six frontal trapezoids, half with sides converging upward and half converging downward, at three degrees of vertical compression. Each $O$ was thus given 12 presentations. Including the triangles he made, in all, 24 matches or judgments of shape-and-slant. There were $30 \mathrm{Os}$ in the experiment.

The standard stimulus in this experiment was, in effect, a sheaf of light rays and there were 12 different ray sheaves employed, six being quadrilateral in cross section and six being triangular. For each of these 12 stimuli a set of comparison stimuli was prepared. These, it will be recalled, were shapes mounted at various slants on a panel. Consider, for example, the ray sheaf illustrated in Fig. 1. Its cross section is a flattened trape- 


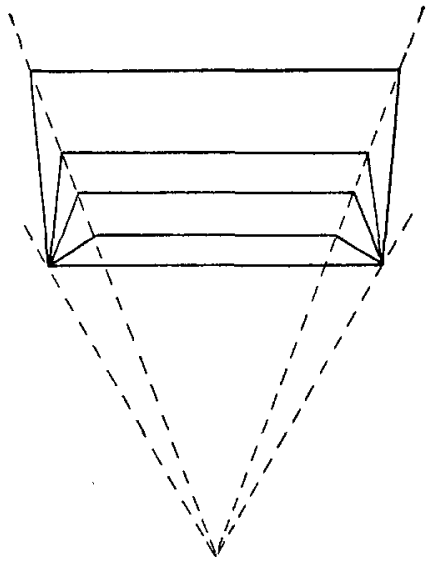

FIG. 1. Schematic view looking down on the sheaf of rays from a rectangle at a $60^{\circ}$ inclination floorwise. Equivalent trapezoids are indicated at lesser inclinations, and one at zero inclination. The shapes constitute a "transformation series." In the experiment there were six such series and, from each, $O$ was shown two standard objects, both the inclined rectangle and the frontal trapezoid.

zoid. The $O$ might perceive a rectangle with a top slanted backward $60^{\circ}$, or either of two trapezoids with tops slanted at lesser amounts, or a still more flattened trapezoid on the frontal plane. This group of quadrilaterals will be called a "transformation series." The set of comparison objects for each stimulus had to include a number of shape-and-slant combinations which were within the relevant transformation series and also some which were outside the series in order to test the hypothesis of an invariant. The latter combinations, if chosen by 0 , would go counter to the hypothesis. The combinations included the cases for which an invariant relation would be most likely to fail.

The appropriate set of comparison objects was mounted on $O$ 's right on a panel illuminated by a low-wattage red lamp. It included from two to four choices which would be consistent with the invariant relation (depending on the degree of slant of the standard) and two choices counter to the invariant.

Each $O$ was seated in the darkroom in front of a screen with an aperture for monocular vision and a biting board. He was shown the comparison objects and was told that they were similar to (but not the same as) the objects he would see through the aperture. He was instructed about the aperture and told that when the shutter was raised he would see a luminous figure at some distance. He was to note both its shape and its slant (since it might be either
TABLE 1

Distribution of Judgments Among Six Comparison Objects in the Quadrilateral Group

\begin{tabular}{|c|c|c|c|}
\hline \multicolumn{2}{|c|}{$\begin{array}{c}\text { Objects Within } \\
\text { Transformation Series }\end{array}$} & \multicolumn{2}{|c|}{$\begin{array}{l}\text { Objects Outside of } \\
\text { Transformation Series }\end{array}$} \\
\hline Judgments & No. & Judgments & No. \\
\hline $\begin{array}{l}\text { 1. Rectangle slanted } \\
\text { 2. Cross-sectional } \\
\text { trap. in frontal } \\
\text { plane } \\
\text { 3. Intermediate } \\
\text { trap, at } 15^{\circ} \\
\text { 4. Intermediate } \\
\text { trap. at } 30^{\circ}\end{array}$ & $\begin{array}{r}34 \\
197 \\
61 \\
4\end{array}$ & $\begin{array}{l}\text { 5. Rectangle in } \\
\text { frontal plane } \\
\text { 6. Cross-sectional } \\
\text { trap. slanted }\end{array}$ & $\begin{array}{r}0 \\
64\end{array}$ \\
\hline $\begin{array}{l}\text { Total number of } \\
\text { judgments within } \\
\text { the transformation } \\
\text { series }\end{array}$ & 296 & $\begin{array}{l}\text { Total number of } \\
\text { judgments outside } \\
\text { of the transforma- } \\
\text { tion series }\end{array}$ & 64 \\
\hline
\end{tabular}

frontal to his eye or inclined) and then turn to the panel and match it for shape and slant. He should check his match once, and would be allowed a third look through the aperture if still uncertain. Each presentation of the standard was for $10 \mathrm{sec}$. but there was no time limit for inspecting the comparisons. The 24 matches made by each $O$ were in random order for the various experimental conditions.

Results.-The questions posed are these. Will the matches be variable within the family of projectively equivalent shapes, or not variable? Will they occur wholly within the family or not? Table 1 , representing the quadrilateral stimuli, shows that

\section{TABLE 2}

Distribution of Judgments Among Six Comparison Objects in the Trlangle Group

\begin{tabular}{|c|c|c|c|}
\hline \multicolumn{2}{|l|}{ Within Invariant } & \multicolumn{2}{|l|}{ Counter to Invariant } \\
\hline Judgments & No. & Judgments & No. \\
\hline $\begin{array}{l}\text { 1. Standard triangle } \\
\text { slanted } \\
\text { 2. Cross-gectional } \\
\text { triangle in frontal } \\
\text { plane } \\
\text { 3. Intermediate tri- } \\
\text { angle at } 15^{\circ} \\
\text { 4. Intermediate tri- } \\
\text { angle at } 30^{\circ}\end{array}$ & $\begin{array}{r}24 \\
279 \\
26 \\
3\end{array}$ & $\begin{array}{l}\text { 5. Standard triangle } \\
\text { in frontal plane } \\
\text { 6. Cross-sectional } \\
\text { triangle slanted }\end{array}$ & $\begin{array}{r}0 \\
28\end{array}$ \\
\hline $\begin{array}{l}\text { Total number of } \\
\text { judgments within } \\
\text { the transformation } \\
\text { series }\end{array}$ & 332 & $\begin{array}{l}\text { Total number of } \\
\text { judgments outside } \\
\text { of the transforma- } \\
\text { tion series }\end{array}$ & 28 \\
\hline
\end{tabular}


the phenomenal shapes perceived were variable. No single perception of form was determined under these reduced stimulus conditions, although the perception of a frontal plane form is predominant. The case is similar for the triangular stimuli in Table 2. The results support the hypothesis that phenomenal shape becomes indeterminate when phenomenal slant is made indeterminate, not the classical view that retinal shape determines phenomenal shape when not interfered with. Doubt is cast on the theory of a "form sense" and on the possibility of reducing perceptions of objects to sensations of form.

In answer to the second question, the tables show 64 and 28 matches, respectively, out of a possible $360(12$ matches for 300 s) which are exceptions to the shape-slant invariance formula. We must conclude that the hypothetical linkage between psychological shape and slant is not rigid. It cannot be asserted that a retinal projection of a given form determines a unique relation of apparent shape to apparent slant but only that there is a tendency to such a relation. The results are analogous to those of Stavrianos (10).

The number of exceptions is greater for the quadrilateral than for the triangular stimuli by $10 \%(C R=4.08$, $P=.01$ ). Moreover the number of exceptions, with both quadrilaterals and triangles, is greater for ceilingwise slant than it is for floorwise slant (CR $=3.08$ and 3.20 , respectively; $P=$ $.01)$. These significant differences suggest that the strength of the connections between impressions of shape and slant depends on the kind of shape investigated and on the direction of its slant. They are consistent with the view that the connections may be learned.

There is apparently a strong pre- sumption to see a shape in the frontal plane, even though it is not wholly determining. In both tables the crosssectional shape is more frequent than all the equivalent slanted shapes taken together. The frontal slant can be considered normal or statistically probable, or conceived as a stable structure in the style of gestalt theory. Similarly a rectangle is normal or stable as compared with trapezoids, but the standard triangle presented is not so as compared with the other triangles in the series. A possible tendency, however, to perceive the rectangle more frequently than the standard triangle (34 and 24 matches, respectively) is not confirmed, since the difference is not significant $(P>$ .10). Comparing the slanted rectangle with the frontal trapezoid in Table 1, both percepts being equally possible, it is evident that the presumption of frontal position wins out in a striking way over the presumption of rectangular shape. Neither a statistical "best bet" theory nor a theory of stable structures provides any ready explanation of the strength of these presumptions, taking the results together. It should be noted that the most probable (or stable) percept of all, the rectangle in the frontal plane, was not once obtained in the whole 360 judgments. It is not clear, however, why this should be so.

\section{EXPERIMENT II}

A different method of testing a consequence of the slant-shape invariance formula was next conceived and tried out. This did not depend on judgments of a luminous shape in the dark but on judgments of a shape against a visible background. The method was based on the expectation that a flat shape of homogeneous color will, when slanted away from a visible textured 
background, assume the slant of its background if binocular disparity and motion perspective are eliminated from stimulation (3, p. 178).

Method.-An isosceles triangle cut out of white cardboard was mounted on a large vertical screen of black painted plasterboard at a $45^{\circ}$ angle of slant outward from the base of the triangle. This was the standard object. It had to be matched with one of two comparison triangles mounted flat on the background, one above and to its left, the other below and to its right. One of these had the same shape as the standard while the other had the shape the standard would have if projected on the background. If the background induces a presumptive error in the impression of slant, this should determine a corresponding error in the impression of shape and $O$ will match the standard with the latter instead of the former. The question is, will a presumptive slant induced by the stimulus situation determine a false judgment of shape?

Three different standard triangles were used, all with 10 -in. bases but with altitudes of 8.75 , 6.75 , and $5 \mathrm{in}$. They will be referred to as $S_{1}$, $S_{2}$, and $S_{3}$, respectively. A set of comparison triangles was prepared, also with 10-in. base but with altitudes of $8.75,6.75,5$, and 3.5 in. They will be referred to as $C_{1}, C_{2}, C_{8}$, and $C_{4}$. A standard and comparison triangle with the same index number are equal in shape (and size). A standard triangle at a $45^{\circ}$ slant is projectively equivalent to the comparison triangle with the next larger index number (approximately).

Each $O$ made three matches of the three standards. He observed the standard and its two comparison objects on their common background through a monocular aperture at a distance of $7 \mathrm{ft}$. The shadow cast by the slanted standard was made invisible by illuminating the objects solely with a circular fluorescent lamp mounted on the far side of the aperture screen and centered on the aperture through which the objects were viewed. Thirty naive $O$ s were used. Each was seated in the darkroom and told that when he applied his eye to the peephole in the screen before him, he would see three triangles mounted on the wall in a diagonal line. He was to judge whether the middle triangle was the same as the one above or the one below it.

Results.-All $O$ s reported seeing the standard triangle as flat against the wall. The effect was compelling and no $O$ voiced any doubt about it. Table 3 shows the results of the ex-
TABLE 3

Comparison Triangles $\mathrm{C}_{1}, \mathrm{C}_{2}$, and $\mathrm{C}_{3}$ Which Were Identified as Equal to Standard Triangles $\mathrm{S}_{1}, \mathrm{~S}_{2}$, AND $\mathrm{S}_{3}$

\begin{tabular}{|c|c|c|c|c|c|c|}
\hline \multirow[b]{2}{*}{$\begin{array}{l}\text { Triangle Presented } \\
\text { Expected Results }\end{array}$} & \multicolumn{3}{|c|}{$\begin{array}{c}\text { Monocular } \\
\text { Vision } \\
\text { (Exp. II) }\end{array}$} & \multicolumn{3}{|c|}{$\begin{array}{l}\text { Binocular } \\
\text { Vision } \\
\text { (Exp. III) }\end{array}$} \\
\hline & $\begin{array}{l}S_{1} \\
C_{2}\end{array}$ & $\begin{array}{l}\mathrm{S}_{2} \\
\mathrm{C}_{z}\end{array}$ & $\underset{\mathbf{S}_{4}}{\mathbf{S}_{\mathbf{s}}}$ & $\begin{array}{l}S_{1} \\
\mathbf{C}_{1}\end{array}$ & $\mathbf{S}_{2}$ & S: \\
\hline $\begin{array}{l}\text { No. of deviations } \\
\text { No. as expected } \\
\text { Per cent as } \\
\text { expected }\end{array}$ & $\begin{array}{r}0 \\
30 \\
100\end{array}$ & $\begin{array}{r}0 \\
30 \\
100\end{array}$ & $\begin{array}{r}0 \\
30 \\
100\end{array}$ & $\begin{array}{r}9 \\
21 \\
70\end{array}$ & $\begin{array}{r}7 \\
23 \\
77\end{array}$ & $\begin{array}{r}7 \\
23 \\
77\end{array}$ \\
\hline
\end{tabular}

periment, along with those of the next experiment in which binocular vision was introduced. The left side of the table indicates that all 30 Os matched the standard with the comparison whose shape was its projection, not with the comparison whose shape was objectively the same. $S_{1}$ was matched with $C_{2}, S_{2}$ with $C_{3}$, and $S_{3}$ with $C_{4}$.

An illusory impression of slant, therefore, goes with a corresponding illusion in the perception of shape, under these conditions. But it may be noted that this experiment is a much less exacting test for the unique linkage hypothesis than the last. It does not show that each of a large number of possible apparent shapes is linked with one of a large number of apparent slants. The method employed, however, could be used in more elaborate experiments and errors could be measured with more precision. The background used was perpendicular to the line of sight and the slant induced on the standard form was frontal. The effect on slant of using a background itself at a slant is not certain. Preliminary experiments suggest that the effect still holds and that illusions of shape can be induced, but the degree of linkage has not been measured. 


\section{EXPERIMENT III}

In the preceding experiments stimulation for the slant of the standard object was eliminated. Constancy of shape probably depends on the presence of such stimulation. The last experiment can be modified to permit it by simply converting the monocular aperture in to a binocular slit.

Method.-The $O$ sees the standard and its two comparisons, as before, but now the middle triangle appears to slant forward from the background. Its edges are increasingly uncrossed disparate from the base to the apex (or decreasingly crossed disparate) and they manifest a slight skew when the head is not held motionless. The texture perspective of its surface, however, is probably invisible since the latter is smooth white cardboard at $7 \mathrm{ft}$. If the slant of the object is correctly seen, the shape should also be correctly seen. The $O$ should then match the standard with the comparison object whose shape is objectively the same, not the one whose shape is projectively the same. In terms of the index numbers, $S_{1}$ should be matched with $C_{1}$, $S_{2}$ with $C_{2}$, and $S_{3}$ with $C_{3}$. The same group of $30 O$ s was used, the binocular matches of this experiment following the monocular ones of the previous experiment. The instructions were the same as before: to judge whether the middle triangle was the same as the one above or below.

Results.-The right side of Table 3 shows that not all $30 \mathrm{Os}$ made the predicted matches. Nine $O$ s matched the tallest triangle $\left(\mathrm{S}_{1}\right)$ with the just shorter one $\left(\mathrm{C}_{2}\right)$, and 7 Os matched the shorter triangles with ones just shorter than themselves $\left(\mathrm{S}_{2}\right.$ with $\mathrm{C}_{3}$, and $\mathrm{S}_{3}$ with $\mathrm{C}_{4}$ ). In general, however, there was a tendency toward constancy of shape. The expected matches predominate.

It cannot be argued that the $O$ s who failed to attain constancy did so because they failed to see the slant of the standard triangle. Several of these $O$ s were asked immediately to reproduce this slant kinesthetically by the method of adjusting a vertical plate, hinged in the middle, with the palm of the hand; they could do so with some accuracy. We must evidently conclude that constancy was imperfect or incomplete in this experiment, an outcome not unusual in experiments of this sort. Why this is so is still unclear.

\section{Discussion}

It has been widely assumed that we perceive the shape of an object in space on the basis of two separate variable impressions, apparent shape and apparent slant. The relation of these phenomenal variables to stimulation and to each other is a difficult and puzzling problem. Their relation to each other under reduced stimulation is the part of the problem most amenable to experimentation. The present experiments are principally of this sort.

The results of these experiments are not conclusive for a theory of constancy, and not even notably consistent with one another. They do cast doubt, however, on the distinction between apparent form as a sensation and apparent slant as a perception. The experimental methods employed, moreover, are promising, and further use of them may prove illuminating. There is a question whether such procedures with reduced stimulation will ever solve the general problem of the perception of objects in the environment. Perhaps the perception of objects is not based on two separate phenomenal variables, shape and slant. If the seeing of the boundaries and the texture of a surface are not distinct phenomena but are instead closely related, the question of shape constancy may be included in the question of slant perception. This seems to the writers the most promising line for future research.

\section{Summary}

The relationship of apparent shape and apparent slant to the perception of the shape of objects in space was studied in three experiments. It was demonstrated in Exp. I that a reduced retinal shape without stimulation for the slant of the surface can induce a whole family of apparent shapes and does not necessarily determine the perpendicular cross-sectional member of the 
family. But there was a strong "presumption" for the frontal shape and, in the quadrilateral family, this won out over the tendency to see a rectangle. As to the linkage between shape and slant within the family of shape-slants, it proved to be by no means perfect. Experiment II showed that, again for a reduced retinal shape, an illusory slant can induce an illusory shape. The phenomenal slant of such an object will tend to be that of the textured background surface, whatever its physical slant may be. Experiment II yielded no exceptions to the linkage hypothesis, but it did not constitute as strict a test. The strictness of the linkage seemed to depend on the kind of shape seen and on the direction of its slant. The linkage may be learned, but a probabalistic theory of the percepts attained does not readily explain the facts. Experiment III showed that constancy of the perceptions of shape appeared when stimulation for slant was provided, but not universally.

\section{REFERENCES}

1. Boring, E. G. Visual perception as invariance. Psychol. Rev., 1952, 59, 141-148.

2. Boring, E. G. The Gibsonian visual field. Psychol. Rev., 1952, 59, 246-247.

3. Grbson, J. J. The perception of the, visual world. Boston: Houghton Mifflin, 1950.
4. Gibson, J. J. What is a form? Psychol. Rev., 1951, 58, 403-412.

5. Gibson, J. J. The visual field and the visual world: a reply to Professor Boring. Psychol. Rev., 1952, 59, 149-151.

6. Helmholtz, H. Physiologicaloptics, Vol. 3. J. P. C. Southall, (Ed.), Optical Soc. Amer., 1925.

7. Holway, A. H., \& Boring, E. G. Determinants of apparent visual size with distance variant. Amer. J. Psychol., 1941, 54, 2137.

8. Kilpatrick, F. P., \& Ittelson, W. H. The size-distance invariance hypothesis. Psychol. Rev., 1953, 60, 223-231.

9. KoFrKa, K. Principles of gestalt psychology. New York: Harcourt Brace, 1935.

10. Langden, J. Further studies in the perception of a changing shape. Quart $J$. exp. Psychol., 1953, 5, 89-107.

11. Stavrianos, B. K. The relation of shapeperception to explicit judgments of inclination. Arch. Psychol., N. Y., 1945, - No. 296.

12. Thouless, R. H. Phenomenal regression to the real object: I. Brit. J. Psychol., 1931, 21, 339-359.

(Received August 12, 1954) 